\title{
Some properties of solutions
}

\section{for an isothermal viscous Cahn-Hilliard equation with inertial term}

\author{
Changchun Liu* and Jiaojiao Wang
}

"Correspondence: liucc@jlu.edu.cn Department of Mathematics, Jilin University, Changchun, China

\begin{abstract}
In this paper, we study the global existence and blow-up of solutions for an isothermal viscous Cahn-Hilliard equation with inertial term, which arises in isothermal fast phase separation processes. Based on the Galerkin method and the compactness theorem, we establish the existence of the global generalized solution. Using a lemma on the ordinary differential inequality of second order, we prove the blow-up of the solution for the initial-boundary problem.
\end{abstract}

MSC: Primary 35L25; secondary 35L35; 35B44

Keywords: Cahn-Hilliard equation with inertial term; Blow-up; Global existence

\section{Introduction}

In this paper, we are concerned with the following initial-boundary problem:

$$
\begin{aligned}
& \delta u_{t t}+u_{t}-k \Delta u_{t}+\Delta^{2} u=\Delta f(u), \quad x \in \Omega, t>0, \\
& \left.u\right|_{\partial \Omega}=0,\left.\quad \Delta u\right|_{\partial \Omega}=0, \quad t \geq 0, \\
& u(x, 0)=\varphi(x), \quad u_{t}(x, 0)=\psi(x), \quad x \in \Omega,
\end{aligned}
$$

where $\Omega \subset \mathbb{R}^{n}(n \leq 3)$ is a bounded domain with smooth boundary, $\delta>0$ is an inertial parameter, $k \geq 0$ is a viscosity coefficient, and $f(s)$ is a given nonlinear function.

Equation (1.1) was proposed in [1] to model rapid spinodal decompositions in a binary alloy. Zheng and Milani [2] proved that the dynamical systems generated by problem (1.1)-(1.3) admit exponential attractors and inertial manifolds. Zheng and Milani [3] show that the dynamical systems admit global attractors and that these global attractors are at least upper-semicontinuous with respect to the vanishing of the perturbation parameter. Gatti et al. [4] considered problem (1.1)-(1.3). Their result is the construction of a robust family of exponential attractors, whose common basins of attraction are the whole phase-space. They [5] also considered the same problem in the three-dimensional setting.

(c) The Author(s) 2018. This article is distributed under the terms of the Creative Commons Attribution 4.0 International License (http://creativecommons.org/licenses/by/4.0/), which permits unrestricted use, distribution, and reproduction in any medium, provided you give appropriate credit to the original author(s) and the source, provide a link to the Creative Commons license, and indicate if changes were made. 
Grasselli et al. [6] studied a differential model describing nonisothermal fast phase separation processes taking place in a three-dimensional bounded domain.

$$
\left\{\begin{array}{l}
(\vartheta+\chi)_{t}+\nabla \cdot \mathbf{q}=0, \\
\sigma \mathbf{q}_{t}+\mathbf{q}=-\nabla \vartheta \\
k \chi_{t t}+\chi_{t}-\Delta\left(-\Delta \chi+\alpha \chi_{t}+\phi(\chi)-\vartheta\right)=0,
\end{array}\right.
$$

where $\sigma \in[0,1]$. This model consists of a viscous Cahn-Hilliard equation characterized by the presence of an inertial term $\chi_{t t}, \chi$ being the order parameter, which is linearly coupled with an evolution equation for the (relative) temperature $\vartheta$.

The blow-up of solutions for the fourth order equation has been intensively studied. Chen and Lu [7] considered the initial-boundary value problem for the nonlinear wave equation

$$
u_{t t}-2 b u_{x x t}+\alpha u_{x x x x}=f\left(u_{x}\right)_{x}
$$

They obtained the blow-up of the solution and the energy decay of the solutions. Wang [8] studied the equation

$$
u_{t t}+\Delta^{2} u+\mu u_{t}+a u=|u|^{p-2} u
$$

He gave necessary and sufficient conditions for global existence and finite time blow-up of solutions. Escudero et al. [9] discussed a fourth order parabolic equation involving the Hessian

$$
u_{t}+\Delta^{2} u=\operatorname{det}\left(D^{2} u\right)=\lambda f
$$

The authors proved the global existence versus blow-up results. Qu and Zhou [10] studied the following:

$$
u_{t}+D^{4} u=|u|^{p-1} u-f_{\Omega}|u|^{p-1} u d x
$$

By using the method of potential wells, they obtained a threshold result of global existence and blow-up for the sign-changing weak solutions and the conditions under which the global solutions become extinct in finite time. In this paper, we consider the global existence and blow-up of solutions for problem (1.1)-(1.3). To prove the blow-up of solutions, we establish a new functional and consider the solution of the Bernoulli type equation. Basing on the required estimates and using a lemma on the ordinary differential inequality of second order, we prove the blow-up of the solution for the initial-boundary problem. The main method is nontrivial because of both the nonlinearity of $\Delta f(u)$ and more delicate estimates which are necessary to overcome some delicate technical points.

The plan of this paper is as follows. In Sect. 2, we prove the existence and uniqueness of the global generalized solution for the initial-boundary value problems (1.1)-(1.3) by the Galerkin method. We also give some sufficient conditions of the blow-up of the solutions for the initial-boundary value problems (1.1)-(1.3) in Sect. 3. Finally, in Sect. 4, we discussed the decay rate of energy. For simplicity, we set $\delta=1$ in this paper. 


\section{Existence of the global solution}

We are going to prove the existence and uniqueness for problems (1.1)-(1.3) by the Galerkin method and the compactness theorem in this section.

Let $y_{i}(x)$ be the orthonormal basis in $L^{2}(\Omega)$ composed of the eigenfunctions of the eigenvalue problem

$$
\left\{\begin{array}{l}
\Delta y+\lambda y=0 \\
\left.y\right|_{\partial \Omega}=0
\end{array}\right.
$$

corresponding to eigenvalue $\lambda_{i}(i=1,2, \ldots)$.

Let

$$
u_{N}(x, t)=\sum_{i=1}^{N} \gamma_{N i}(t) y_{i}(x)
$$

be the Galerkin approximate solution for problem (1.1)-(1.3), where $\gamma_{N i}(t)$ are the undetermined functions and $N$ is a natural number. Suppose that the initial value functions $\varphi(x)$ may be expressed as

$$
\varphi(x)=\sum_{i=1}^{\infty} \mu_{i} y_{i}(x), \quad \psi(x)=\sum_{i=1}^{\infty} v_{i} y_{i}(x),
$$

where $\mu_{i}$ and $v_{i}(i=1,2, \ldots)$ are constants.

Substituting the approximate solution $u_{N}(x, t)$ into Eq. (1.1), multiplying both sides by $y_{s}(x)$, we obtain

$$
\left(u_{N t t}+u_{N t}-k \Delta u_{N t}+\Delta^{2} u_{N}, y_{s}\right)=\left(\Delta f\left(u_{N}\right), y_{s}\right), \quad s=1,2, \ldots, N,
$$

where $(\cdot, \cdot)$ denotes the inner product of $L^{2}(\Omega)$.

Substituting the approximate solution $u_{N}(x, t)$ and the approximations

$$
\varphi_{N}(x)=\sum_{i=1}^{N} \mu_{i} y_{i}(x), \quad \psi_{N}(x)=\sum_{i=1}^{N} v_{i} y_{i}(x)
$$

of the initial value functions into the initial condition (1.3), we get

$$
\gamma_{N s}(0)=\mu_{s}, \quad \dot{\gamma}_{N s}(0)=v_{s}, \quad s=1,2, \ldots, N,
$$

where $\dot{\gamma}_{N s}(t)=\frac{d}{d t} \gamma_{N s}(t)$.

In order to prove the existence of the global generalized solution for problem (1.1)-(1.3), we make a series of estimations for the approximate solution $u_{N}(x, t)$.

Lemma 2.1 Suppose that $\varphi \in H^{2}(\Omega)$ and $\psi \in L^{2}(\Omega)$ satisfy the boundary condition (1.2), $f \in C^{1}(R), 0 \leq F(s)=\int_{0}^{s} f(\eta) d \eta$, and $\left|f^{\prime}(s)\right| \leq C_{1}|s|^{2}+C_{2}$, where $C_{1}>0, C_{2}>0$ are constants. Then the following estimate holds:

$$
\left\|u_{N}(\cdot, t)\right\|_{H^{2}}^{2}+\left\|u_{N t}(\cdot, t)\right\|^{2} \leq C, \quad t \in[0, T]
$$

where and in the sequel $C>0$ is a constant which only depends on $T$. 
Proof Let $w_{n}$ be the unique solution of the problem

$$
\begin{aligned}
& \Delta w_{n}=u_{n}, \\
& \left.w_{n}\right|_{\partial \Omega}=0 .
\end{aligned}
$$

Substituting the approximate solution $u_{N}(x, t)$ into Eq. (1.1), multiplying both sides by $2 w_{N t}$, we obtain

$$
\left(u_{N t t}+u_{N t}-k \Delta u_{N t}+\Delta^{2} u_{N}, 2 w_{N t}\right)=\left(\Delta f\left(u_{N}\right), 2 w_{N t}\right) .
$$

Integrating by parts with respect to $x$ on $\Omega$, we have

$$
\begin{aligned}
& \frac{d}{d t}\left[\left\|\nabla w_{N t}(\cdot, t)\right\|^{2}+\left\|\nabla u_{N}(\cdot, t)\right\|^{2}+2 \int_{0}^{1} F\left(u_{n}\right) d x\right] \\
& \quad+2\left\|\nabla w_{N t}(\cdot, t)\right\|^{2}+2 k\left\|u_{N t}(\cdot, t)\right\|^{2} \leq 0 .
\end{aligned}
$$

Hence, we know

$$
\begin{gathered}
\left\|\nabla w_{N t}\right\| \leq C, \\
\left\|\nabla u_{N}\right\| \leq C .
\end{gathered}
$$

By the Sobolev imbedding theorem, it follows from (2.7) and (2.8) that

$$
\begin{aligned}
& \left\|u_{N}\right\|_{L^{q}} \leq C, \quad \text { for any } q<\infty \quad(n=2), \\
& \left\|u_{N}\right\|_{L^{6}} \leq C \quad(n=3) .
\end{aligned}
$$

Multiplying both sides of (2.4) by $2 \gamma_{N s t}(t)$, summing up for $s=1,2, \ldots, N$, we have

$$
\left(u_{N t t}+u_{N t}-k \Delta u_{N t}+\Delta^{2} u_{N}, 2 u_{N t}\right)=\left(\Delta f\left(u_{N}\right), 2 u_{N t}\right) .
$$

Integrating by parts with respect to $x$ on $\Omega$, we get

$$
\begin{aligned}
& \frac{d}{d t}\left[\left\|u_{N t}(\cdot, t)\right\|^{2}+\left\|\Delta u_{N}(\cdot, t)\right\|^{2}\right]+2\left\|u_{N t}\right\|^{2}+2 k\left\|\nabla u_{N t}(\cdot, t)\right\|^{2} \\
& \quad \leq \frac{1}{4 k}\left\|f^{\prime}\left(u_{N}\right) \nabla u_{N}(\cdot, t)\right\|^{2}+k\left\|\nabla u_{N t}(\cdot, t)\right\|^{2} .
\end{aligned}
$$

By $\left|f^{\prime}(s)\right| \leq C_{1}|u|^{2}+C_{2}$, hence

$$
\begin{aligned}
& \frac{d}{d t}\left[\left\|u_{N t}(\cdot, t)\right\|^{2}+\left\|\Delta u_{N}(\cdot, t)\right\|^{2}\right]+2\left\|u_{N t}\right\|^{2}+k\left\|\nabla u_{N t}(\cdot, t)\right\|^{2} \\
& \quad \leq C\left\|u_{N}\right\|_{\infty}^{4}+C .
\end{aligned}
$$

On the other hand, by the Gagliardo-Nirenberg inequality, (2.9), and (2.10), we see

$$
\begin{aligned}
& \|u\|_{\infty} \leq C\|\Delta u\|^{a}\|u\|_{q}^{1-a} \leq C\|\Delta u\|^{a}, \quad a=\frac{2}{q+2}(n=2), \\
& \|u\|_{\infty} \leq C\|\Delta u\|^{1 / 2}\|u\|_{6}^{1 / 2} \leq C\|\Delta u\|^{1 / 2} \quad(n=3) .
\end{aligned}
$$


Therefore, by (2.11),

$$
\begin{aligned}
& \frac{d}{d t}\left[\left\|u_{N t}(\cdot, t)\right\|^{2}+\left\|\Delta u_{N}(\cdot, t)\right\|^{2}\right]+2\left\|u_{N t}\right\|^{2}+k\left\|\nabla u_{N t}(\cdot, t)\right\|^{2} \\
& \quad \leq C\left\|\Delta u_{N}\right\|^{2}+C
\end{aligned}
$$

Then, integrating (2.12) on $[0, t]$ and using the Gronwall inequality, we deduce

$$
\left\|u_{N}(\cdot, t)\right\|_{H^{2}}^{2}+\left\|u_{N t}(\cdot, t)\right\|^{2} \leq C e^{T}\left(\|\varphi\|_{H^{2}}^{2}+\|\psi\|^{2}+1\right), \quad t \in[0, T] .
$$

Immediately, we get (2.6) from (2.13). The proof is completed.

Lemma 2.2 Suppose that the conditions of Lemma 2.1 hold. If $f \in C^{3}(R), \varphi \in H^{4}(\Omega)$, $\psi \in H^{2}(\Omega)$, and $\left|f^{\prime \prime}(s)\right| \leq C_{3}|s|+C_{4},\left|f^{\prime \prime \prime}(s)\right| \leq C$, then the approximate solution for problem (1.1)-(1.3) satisfies the following estimate:

$$
\left\|u_{N}(\cdot, t)\right\|_{H^{4}}^{2}+\left\|u_{N t}(\cdot, t)\right\|_{H^{2}}^{2}+\left\|u_{N t t}(\cdot, t)\right\|^{2} \leq C(T), \quad 0 \leq t \leq T .
$$

Proof Multiplying both sides of (2.4) by $2 \lambda_{s}^{2} \gamma_{N s t}(t)$, summing up for $s=1,2, \ldots, N$, we have

$$
\left(u_{N t t}+u_{N t}-k \Delta u_{N t}+\Delta^{2} u_{N}, 2 \Delta^{2} u_{N t}\right)=\left(\Delta f\left(u_{N}\right), 2 \Delta^{2} u_{N t}\right) .
$$

Integrating by parts with respect to $x$, we get

$$
\begin{aligned}
& \frac{d}{d t}\left(\left\|\Delta u_{N t}(\cdot, t)\right\|^{2}+\left\|\Delta^{2} u_{N}(\cdot, t)\right\|^{2}\right)+2\left\|\Delta u_{N t}(\cdot, t)\right\|^{2}+2 k\left\|\nabla \Delta u_{N t}(\cdot, t)\right\|^{2} \\
& \quad=2 \int_{\Omega} \Delta f\left(u_{N}\right) \cdot \Delta^{2} u_{N t} d x
\end{aligned}
$$

On the other hand, we know

$$
2 \int_{\Omega} \Delta f\left(u_{N}\right) \cdot \Delta^{2} u_{N t} d x=-2 \int_{\Omega} \nabla \Delta f\left(u_{N}\right) \cdot \nabla \Delta u_{N t} d x
$$

and

$$
\nabla \Delta f\left(u_{N}\right)=f^{\prime \prime \prime}\left(u_{N}\right)\left|\nabla u_{N}\right|^{2} \nabla u_{N}+3 f^{\prime \prime}\left(u_{N}\right) \nabla u_{N} \Delta u_{N}+f^{\prime}\left(u_{N}\right) \nabla \Delta u_{N} .
$$

By (2.13), we know that $\|u\|_{\infty} \leq C$, hence

$$
\begin{aligned}
& 2 \int_{\Omega} \Delta f\left(u_{N}\right) \cdot \Delta^{2} u_{N t} d x \\
& \quad \leq C\left(\left\|\left|\nabla u_{N}\right|^{3}\right\|^{2}+\left\|\nabla u_{N} \Delta u_{N}\right\|^{2}+\left\|\nabla \Delta u_{N}\right\|^{2}\right)+k\left\|\nabla \Delta u_{N t}(\cdot, t)\right\|^{2} .
\end{aligned}
$$

Thus,

$$
\begin{aligned}
& \frac{d}{d t}\left(\left\|\Delta u_{N t}(\cdot, t)\right\|^{2}+\left\|\Delta^{2} u_{N}(\cdot, t)\right\|^{2}\right)+2\left\|\Delta u_{N t}(\cdot, t)\right\|^{2}+k\left\|\nabla \Delta u_{N t}(\cdot, t)\right\|^{2} \\
& \quad \leq C\left(\left\|\nabla u_{N}(\cdot, t)\right\|_{L^{6}}^{2}+\left\|\nabla u_{N}(\cdot, t)\right\|_{L^{4}}^{2}\left\|\Delta u_{N}(\cdot, t)\right\|_{L^{4}}^{2}+\left\|\nabla \Delta u_{N}(\cdot, t)\right\|^{2}\right) .
\end{aligned}
$$


By (2.13) and the Sobolev imbedding theorem, we see that

$$
\begin{aligned}
& \left\|\nabla u_{N}\right\|_{L^{q}} \leq C, \quad \text { for any } q<\infty(n=2), \\
& \left\|\nabla u_{N}\right\|_{L^{6}} \leq C \quad(n=3) .
\end{aligned}
$$

Using the Gagliardo-Nirenberg inequality, we conclude

$$
\begin{aligned}
\left\|\Delta u_{N}(\cdot, t)\right\|_{L^{4}}^{2} & \leq C\left\|\Delta^{2} u_{N}(\cdot, t)\right\|^{a}\left\|\nabla u_{N}(\cdot, t)\right\|_{L^{q}}^{1-a} \\
& \leq C\left\|\Delta^{2} u_{N}(\cdot, t)\right\|+C, \quad \text { where } a=\frac{q+4}{4+4 q}(n=2), \\
\left\|\Delta u_{N}(\cdot, t)\right\|_{L^{4}}^{2} & \leq C\left\|\Delta^{2} u_{N}(\cdot, t)\right\|^{3 / 8}\left\|\nabla u_{N}(\cdot, t)\right\|_{L^{6}}^{5 / 8} \leq C\left\|\Delta^{2} u_{N}(\cdot, t)\right\|+C \quad(n=3) .
\end{aligned}
$$

On the other hand, by boundary conditions (1.2), we obtain

$$
\left\|\nabla \Delta u_{N}\right\|^{2} \leq C\left\|\Delta^{2} u_{N}\right\|^{2}
$$

Substituting the above inequalities into (2.15), we get

$$
\frac{d}{d t}\left(\left\|\Delta u_{N t}(\cdot, t)\right\|^{2}+\left\|\Delta^{2} u_{N}(\cdot, t)\right\|^{2}\right) \leq C(T)+\left\|\Delta^{2} u_{N}(\cdot, t)\right\|^{2}
$$

Integrating the above inequality, and using the Gronwall inequality, we have

$$
\left\|\Delta u_{N t}(\cdot, t)\right\|^{2}+\left\|\Delta^{2} u_{N}(\cdot, t)\right\|^{2} \leq C(T)\left(\|\varphi\|_{H^{4}}^{2}+\|\psi\|_{H^{2}}^{2}+1\right), \quad t \in[0, T]
$$

Similarly, multiplying both sides of (2.4) by $\gamma_{N s t t}(t)$, summing up for $s=1,2, \ldots, N$, we deduce

$$
\left(u_{N t t}+u_{N t}-k \Delta u_{N t}+\Delta^{2} u_{N}, u_{N t t}\right)=\left(\Delta f\left(u_{N}\right), u_{N t t}\right)
$$

Integrating by parts with respect to $x$ and using the Cauchy inequality, we have

$$
\begin{aligned}
& \left\|u_{N t t}(\cdot, t)\right\|_{L^{2}}^{2} \\
& =-\int_{\Omega} u_{N t} u_{N t t} d x+k \int_{\Omega} \Delta u_{N t} u_{N t t} d x-\int_{\Omega} \Delta^{2} u_{N} u_{N t t} d x+\int_{\Omega} \Delta f\left(u_{N}\right) u_{N t t} d x \\
& \leq 2\left\|u_{N t}(\cdot, t)\right\|^{2}+2 k^{2}\left\|\Delta u_{N t}(\cdot, t)\right\|^{2}+2\left\|\Delta^{2} u_{N}(\cdot, t)\right\|^{2} \\
& \quad+2\left\|\Delta f\left(u_{N}\right)\right\|^{2}+\frac{1}{2}\left\|u_{N t t}(\cdot, t)\right\|^{2} .
\end{aligned}
$$

Therefore, we conclude

$$
\left\|u_{N t t}(\cdot, t)\right\|_{L^{2}}^{2} \leq C(T), \quad t \in[0, T]
$$

Immediately, we get (2.14) from (2.16) and (2.17). This completes the proof. 
Theorem 2.1 Suppose that $\varphi \in H^{4}(\Omega)$ and $\psi \in H^{2}(\Omega)$ satisfy the boundary conditions $(1.2), f \in C^{3}(R), 0 \leq F(s)=\int_{0}^{s} f(\eta) d \eta,\left|f^{\prime}(s)\right| \leq C_{1}|s|^{2}+C_{2},\left|f^{\prime \prime}(s)\right| \leq C_{3}|s|+C_{4}$, and $\left|f^{\prime \prime \prime}(s)\right| \leq$ $C$. Then problem (1.1)-(1.3) has a unique global generalized solution

$$
u \in C\left([0, T] ; H^{4}(\Omega)\right) \cap C^{1}\left([0, T] ; H^{2}(\Omega)\right) \cap C^{2}\left([0, T] ; L^{2}(\Omega)\right) .
$$

Proof From (2.14) we know that $u_{N} \in C\left([0, T] ; H^{4}(\Omega)\right), u_{N t} \in C\left([0, T] ; H^{2}(\Omega)\right), u_{N t t} \in$ $C\left([0, T] ; L^{2}(\Omega)\right)$. Using the Sobolev imbedding theorem, we have $D^{k} u_{N} \in C([0, T] \times \Omega)$, $0 \leq k \leq 2$. It follows from the above two relations and the Ascoli-Arzela theorem that there exist a function $u(x, t)$ and a subsequence of $u_{N}(x, t)$, still denoted by $u_{N}(x, t)$, such that as $N \rightarrow \infty, u_{N}(x, t)$ uniformly converges to $u(x, t)$ in $[0, T] \times \Omega$. The corresponding subsequence of $\Delta u_{N}(x, t)$ also uniformly converges to $\Delta u(x, t)$ in $[0, T] \times \Omega$. According to the compactness theorem, the subsequence $D^{k} u_{N}(x, t)(0 \leq k \leq 4), D^{k} u_{N t}(x, t)(0 \leq k \leq 2)$, and $u_{N t t}(x, t)$ weakly converge to $D^{k} u(x, t)(0 \leq k \leq 4), D^{k} u_{t}(x, t)(0 \leq k \leq 2)$, and $u_{t t}(x, t)$ in $L^{2}([0, T] \times \Omega)$, respectively. Hence, we know that $u(x, t)$ satisfies $(2.18)$. Therefore $u(x, t)$ is the generalized solution for problem (1.1)-(1.3). It is easy to prove the uniqueness of the solutions for problem (1.1)-(1.3). This completes the proof of the theorem.

\section{Blow-up of solutions}

In the previous sections, we have seen that the solution of problem (1.1)-(1.3) is globally existent, provided that $F(s) \geq 0$. In this section, we will prove the blow-up of the solution for $F(s)<0$. For this purpose, we need the following lemma.

Lemma 3.1 ([7]) Assume that $u^{\prime}=G(t, u), v^{\prime} \geq G(t, v), G \in C([0, \infty) \times(-\infty, \infty))$, and $u\left(t_{0}\right)=v\left(t_{0}\right), t_{0} \geq 0$, then when $t \geq t_{0}, v(t) \geq u(t)$, where $u^{\prime}=\frac{d}{d t} u(t)$.

Let $w$ be the unique solution of the problem

$$
\begin{gathered}
\Delta w=u, \\
\left.w\right|_{\partial \Omega}=0 .
\end{gathered}
$$

We have the following theorem.

\section{Theorem 3.1 Suppose that}

(1) $f(s) s \leq \gamma F(s), F(s) \leq-\alpha|s|^{p+1}$, where $F(s)=\int_{0}^{s} f(u) d u, \gamma>2, \alpha>0$, and $p>1$ are constants.

(2) $\varphi \in H^{4}(\Omega), \psi \in H^{2}(\Omega)$ and

$$
\begin{aligned}
E(0) & =\left\|\nabla w_{t}(0)\right\|^{2}+\|\nabla \varphi\|^{2}+2 \int_{\Omega} F(\varphi(x)) d x \\
& \leq \frac{-2^{\frac{2 p}{p-1}}}{\left(\frac{\alpha(\gamma-2)}{p+3}\right)^{\frac{2}{p-1}}\left(1-e^{-\frac{p-1}{4}}\right)^{\frac{4}{p-1}}}<0,
\end{aligned}
$$

then the generalized solution $u(x, t)$ of problem (1.1)-(1.3) blows up in finite time, i.e.,

$$
\|\nabla w\|^{2}+\int_{0}^{t}\|u(\cdot, \tau)\|^{2} d \tau+\int_{0}^{t}\|\nabla w(\cdot, \tau)\|^{2} d \tau+\int_{0}^{t} \int_{0}^{\tau}\|u\|^{2} d s d \tau \rightarrow \infty, \quad \text { as } t \rightarrow T^{*} .
$$


Proof Let

$$
\begin{aligned}
E(t)= & \int_{\Omega}\left|\nabla w_{t}\right|^{2} d x+2 k \int_{0}^{t} \int_{\Omega}\left|u_{\tau}(x, \tau)\right|^{2} d x d \tau+\int_{\Omega}|\nabla u|^{2} d x \\
& +2 \int_{\Omega} F(u) d x+2 \int_{0}^{t} \int_{\Omega}\left|\nabla w_{\tau}(x, \tau)\right|^{2} d x d \tau .
\end{aligned}
$$

A simple calculation shows that

$$
\begin{aligned}
\frac{d E(t)}{d t}= & 2 \int_{\Omega} \nabla w_{t} \nabla w_{t t} d x+2 k \int_{\Omega}\left|u_{t}\right|^{2} d x+2 \int_{\Omega} \nabla u \nabla u_{t} d x \\
& +2 \int_{\Omega} f(u) u_{t} d x+2 \int_{\Omega}\left|\nabla w_{t}\right|^{2} d x \\
= & -2 \int_{\Omega} u_{t} w_{t t} d x+2 k \int_{\Omega} u_{t} \Delta w_{t} d x-2 \int_{\Omega} \Delta u u_{t} d x \\
& +2 \int_{\Omega} f(u) u_{t} d x-2 \int_{\Omega} w_{t} u_{t} d x \\
= & -2 \int_{\Omega}\left[w_{t t}-k \Delta w_{t}+\Delta u-f(u)+w_{t}\right] u_{t} d x .
\end{aligned}
$$

Noticing equation (1.1), we know that

$$
\frac{d E(t)}{d t}=\int_{\Omega} \nabla\left[w_{t t}-k \Delta w_{t}+\Delta u-f(u)+w_{t}\right]^{2} d x=0,
$$

which implies

$$
E(t)=E(0), \quad t>0
$$

Moreover, we easily see

$$
\begin{aligned}
\gamma \int_{\Omega} F(u) d x= & 2 E(0)-2\left\|\nabla w_{t}\right\|^{2}-4 k \int_{0}^{t}\left\|u_{\tau}\right\|^{2} d \tau-2\|\nabla u\|^{2} \\
& -2 \int_{0}^{t}\left|\nabla w_{t}\right|^{2} d \tau+(\gamma-2) \int_{\Omega} F(u) d x .
\end{aligned}
$$

Now, we define

$$
H(t)=\|\nabla w\|^{2}+\int_{0}^{t}\|u(\cdot, \tau)\|^{2} d \tau+\int_{0}^{t}\|\nabla w(\cdot, \tau)\|^{2} d \tau+\int_{0}^{t} \int_{0}^{\tau}\|u\|^{2} d s d \tau .
$$

It is obvious that

$$
\begin{aligned}
\frac{d H(t)}{d t}= & 2 \int_{\Omega} \nabla w \nabla w_{t} d x+\int_{\Omega}|u(\cdot, t)|^{2} d x \\
& +\int_{\Omega}|\nabla w(\cdot, t)|^{2} d x+\int_{0}^{t} \int_{\Omega}|u|^{2} d x d \tau .
\end{aligned}
$$


Further, we have

$$
\begin{aligned}
\frac{d^{2} H(t)}{d t^{2}}= & 2 \int_{\Omega}\left[\left|\nabla w_{t}\right|^{2}-w u_{t t}+u(\cdot, t) u_{t}+\nabla w \nabla w_{t}+\frac{1}{2}|u|^{2}\right] d x \\
= & 2 \int_{\Omega}\left[\left|\nabla w_{t}\right|^{2}-w u_{t t}+w(\cdot, t) \Delta u_{t}+\nabla w \nabla w_{t}+\frac{1}{2}|u|^{2}\right] d x \\
= & 2 \int_{\Omega}\left[\left|\nabla w_{t}\right|^{2}+w\left(u_{t}+\Delta^{2} u-\Delta f(u)\right)+\nabla w \nabla w_{t}+\frac{1}{2}|u|^{2}\right] d x \\
= & 2 \int_{\Omega}\left[\left|\nabla w_{t}\right|^{2}-\nabla w \nabla w_{t}-|\nabla u|^{2}-f(u) u+\nabla w \nabla w_{t}+\frac{1}{2}|u|^{2}\right] d x \\
\geq & 2\left\|\nabla w_{t}\right\|^{2}-2\|\nabla u\|^{2}-2 \gamma \int_{\Omega} F(u) d x+\|u\|^{2} \\
\geq & 2\left\|\nabla w_{t}\right\|^{2}-2\|\nabla u\|^{2}+\|u\|^{2}-2 E(0)+2\left\|\nabla u_{t}\right\|^{2}+2 \int_{0}^{t}\left\|u_{t}\right\|^{2} d \tau \\
& +2\|\nabla u\|^{2}+2 \int_{0}^{t}\left|\nabla w_{t}\right|^{2} d \tau-2(\gamma-2) \int_{\Omega} F(u) d x \\
\geq & 2\left\|\nabla w_{t}\right\|^{2}+\|u\|^{2}-2 E(0)-2(\gamma-2) \int_{\Omega} F(u) d x+2\left\|\nabla u_{t}\right\|^{2}>0 .
\end{aligned}
$$

Integrating (3.5), we conclude that

$$
H^{\prime}(t) \geq-2 E(0) t-2(\gamma-2) \int_{0}^{t} \int_{\Omega} F(u(x, \tau)) d x d \tau+H^{\prime}(0) .
$$

Integrating (3.6), we deduce

$$
H(t) \geq-2 E(0) t^{2}-2(\gamma-2) \int_{0}^{t} \int_{0}^{\tau} \int_{\Omega} F(u(x, s)) d x d s d \tau+H^{\prime}(0) t+H(0) .
$$

Combining (3.5, (3.6) with (3.7), we derive

$$
\begin{aligned}
H^{\prime \prime}(t) & +H^{\prime}(t)+H(t) \\
\geq & 2 \alpha(\gamma-2)\left[\int_{\Omega}|u|^{p+1} d x+\int_{0}^{t} \int_{\Omega}|u(x, \tau)|^{p+1} d x d \tau\right. \\
& \left.+\int_{0}^{t} \int_{0}^{\tau} \int_{\Omega}|u(x, s)|^{p+1} d x d s d \tau\right]+\|u\|^{2}-2 E(0)\left(1+t+\frac{t^{2}}{2}\right) \\
& +H^{\prime}(0)(1+t)+H(0)+2\left\|\nabla w_{t}\right\|^{2}+2\left\|\nabla u_{t}\right\|^{2} .
\end{aligned}
$$

Substituting (3.4) into (3.8), we get

$$
\begin{aligned}
H^{\prime \prime}(t) & +2 \int_{\Omega} \nabla w \nabla w_{t} d x+\int_{\Omega}|u(\cdot, t)|^{2} d x+\int_{\Omega}|\nabla w(\cdot, t)|^{2} d x \\
& +\int_{0}^{t} \int_{\Omega}|u|^{2} d x d \tau+H(t) \\
\geq 2 & \alpha(\gamma-2)\left[\int_{\Omega}|u|^{p+1} d x+\int_{0}^{t} \int_{\Omega}|u(x, \tau)|^{p+1} d x d \tau\right.
\end{aligned}
$$




$$
\begin{aligned}
& \left.+\int_{0}^{t} \int_{0}^{\tau} \int_{\Omega}|u(x, s)|^{p+1} d x d s d \tau\right]+\|u\|^{2}-2 E(0)\left(1+t+\frac{t^{2}}{2}\right) \\
& +H^{\prime}(0)(1+t)+H(0)+2\left\|\nabla w_{t}\right\|^{2}+2\left\|\nabla u_{t}\right\|^{2}
\end{aligned}
$$

Recalling $H^{\prime \prime}(t)>0, H(t) \geq 0$, and

$$
2 \int_{\Omega} \nabla w \nabla w_{t} d x \leq\|\nabla w\|^{2}+\left\|\nabla w_{t}\right\|^{2}
$$

therefore, from (3.9) we obtain

$$
\begin{aligned}
& H^{\prime \prime}(t)+H(t) \\
& \geq \alpha(\gamma-2)\left[\int_{\Omega}|u|^{p+1} d x+\int_{0}^{t} \int_{\Omega}|u(x, \tau)|^{p+1} d x d \tau\right. \\
& \left.\quad+\int_{0}^{t} \int_{0}^{\tau} \int_{\Omega}|u(x, s)|^{p+1} d x d s d \tau\right] \\
& \quad-E(0)\left(1+t+\frac{t^{2}}{2}\right)+\frac{1}{2} H^{\prime}(0)(1+t)+\frac{1}{2} H(0) .
\end{aligned}
$$

On the other hand, the Hölder inequality implies that

$$
\begin{aligned}
& |\Omega|^{\frac{p-1}{2}} \int_{\Omega}|u|^{p+1} d x \geq\|u\|^{p+1}, \\
& \int_{0}^{t} \int_{\Omega}|u|^{2} d x d \tau \leq t^{\frac{p-1}{p+1}}|\Omega|^{\frac{p-1}{p+1}}\left(\int_{0}^{t} \int_{\Omega}|u|^{p+1} d x d \tau\right)^{\frac{2}{p+1}}, \\
& \int_{0}^{t} \int_{0}^{\tau} \int_{\Omega}|u|^{2} d x d s d \tau \leq\left(\frac{t^{2}}{2}\right)^{\frac{p-1}{p+1}}|\Omega|^{\frac{p-1}{p+1}}\left(\int_{0}^{t} \int_{0}^{\tau} \int_{\Omega}|u|^{p+1} d x d s d \tau\right)^{\frac{2}{p+1}} .
\end{aligned}
$$

Thus

$$
\begin{aligned}
& \int_{0}^{t} \int_{\Omega}|u|^{p+1} d x d \tau \geq t^{\frac{1-p}{2}}|\Omega|^{\frac{1-p}{2}}\left(\int_{0}^{t} \int_{\Omega}|u|^{2} d x d \tau\right)^{\frac{p+1}{2}} \\
& \int_{0}^{t} \int_{0}^{\tau} \int_{\Omega}|u|^{p+1} d x d s d \tau \geq 2^{\frac{p-1}{2}} t^{1-p}\left(\int_{0}^{t} \int_{0}^{\tau} \int_{\Omega}|u|^{2} d x d s d \tau\right)^{\frac{p+1}{2}}
\end{aligned}
$$

Substituting the above inequalities into (3.10), and by the fact $(x+y+z)^{n} \leq 2^{2(n-1)}\left(x^{n}+y^{n}+\right.$ $\left.z^{n}\right), x, y, z>0, n>1$, we know that

$$
\begin{aligned}
& H^{\prime \prime}(t)+H(t) \\
& \geq \alpha(\gamma-2)|\Omega|^{\frac{1-p}{2}}\left[\|u\|^{p+1}+t^{\frac{1-p}{2}}\left(\int_{0}^{t} \int_{\Omega}|u|^{2} d x d \tau\right)^{\frac{p+1}{2}}\right. \\
& \left.\quad+2^{\frac{p-1}{2}} t^{1-p}\left(\int_{0}^{t} \int_{0}^{\tau} \int_{\Omega}|u|^{2} d x d s d \tau\right)^{\frac{p+1}{2}}\right] \\
& \quad-E(0)\left(1+t+\frac{t^{2}}{2}\right)+\frac{1}{2} H^{\prime}(0)(1+t)+\frac{1}{2} H(0)
\end{aligned}
$$




$$
\begin{aligned}
\geq & \alpha(\gamma-2)|\Omega|^{\frac{1-p}{2}} 2^{\frac{1}{2}-\frac{3}{2} p} t^{1-p} H^{\frac{p+1}{2}}-F(0)\left(1+t+\frac{t^{2}}{2}\right) \\
& +\frac{1}{2} H^{\prime}(0)(1+t)+\frac{1}{2} H(0), \quad t \geq 1 .
\end{aligned}
$$

In addition, note from (3.6) and (3.7) that $H^{\prime}(t) \rightarrow+\infty$ and $H(t) \rightarrow+\infty$ as $t \rightarrow \infty$. Therefore, we see that there is $t_{0} \geq 1$ such that when $t \geq t_{0}, H^{\prime}(t)>0$ and $H(t)>0$. Multiplying (3.11) by $2 H^{\prime}(t)$, we get

$$
\frac{d}{d t}\left[H^{\prime 2}+H^{2}\right] \geq M t^{1-p} \frac{d}{d t} H^{\frac{p+3}{2}}+I(t), \quad t \geq t_{0}
$$

where

$$
\begin{aligned}
& M=\frac{\alpha(\gamma-2)|\Omega|^{\frac{1-p}{2}} 2^{\frac{1}{2}-\frac{3}{2} p}}{p+3}, \\
& I(t)=\left[-4 F(0) t+2 H^{\prime}(0)\right]\left[-E(0)\left(1+t+\frac{t^{2}}{2}\right)+\frac{1}{2} H^{\prime}(0)(1+t)+\frac{1}{2} H(0)\right] .
\end{aligned}
$$

It follows from (3.12) that

$$
\frac{d}{d t}\left[t^{p-1}\left(H^{\prime 2}+H^{2}\right)-M H^{\frac{p+3}{2}}\right] \geq t^{p-1} I(t), \quad t \geq t_{0}
$$

Integrating the above inequality over $\left(t_{0}, t\right)$, we easily see

$$
\begin{aligned}
& t^{p-1}\left(H^{\prime 2}+H^{2}\right)-M H^{\frac{n+3}{2}} \\
& \quad \geq \int_{t_{0}}^{t} \tau^{p-1} I(\tau) d \tau+t_{0}^{p-1}\left(H^{\prime 2}\left(t_{0}\right)+H^{2}\left(t_{0}\right)\right)-M H^{\frac{n+3}{2}}\left(t_{0}\right), \quad t \geq t_{0} .
\end{aligned}
$$

Note that when $t \rightarrow \infty$, the right-hand side of (3.13) approaches positive infinity, hence, there is $t_{1}>t_{0}$ such that when $t \geq t_{1}$, the right-hand side of (3.13) is larger than or equal to zero. We thus have

$$
t^{p-1}\left(H^{\prime}+H\right)^{2} \geq t^{p-1}\left(H^{\prime 2}+H^{2}\right) \geq M H^{\frac{p+3}{2}}(t), \quad t \geq t_{0}
$$

that is,

$$
H^{\prime}+H \geq t^{\frac{1-p}{2}} M_{1} H^{\frac{p+3}{4}}(t), \quad t \geq t_{0}
$$

where $M_{1}=M^{1 / 2}$.

Now, we consider the initial value problem of the ordinary differential equation

$$
\begin{aligned}
& S^{\prime}(t)+S(t)=M_{1} t^{\frac{1-p}{2}}(S(t))^{\frac{p+3}{4}}(t), \\
& S\left(t_{1}\right)=H\left(t_{1}\right) .
\end{aligned}
$$


Therefore, we conclude that

$$
\begin{aligned}
S(t) & =e^{-\left(t-t_{1}\right)}\left[H^{\frac{1-p}{4}}\left(t_{1}\right)-\frac{M_{1}(p-1)}{4} \int_{t_{1}}^{t} \tau^{\frac{1-p}{2}} e^{-\frac{p-1}{4}\left(\tau-t_{1}\right)} d \tau\right] \\
& \equiv e^{-\left(t-t_{1}\right)} H\left(t_{1}\right) J^{\frac{4}{1-p}}(t),
\end{aligned}
$$

where

$$
J(t)=1-\frac{M_{1}(p-1)}{4} H^{\frac{p-1}{4}}\left(t_{1}\right) \int_{t_{1}}^{t} \tau^{\frac{1-p}{2}} e^{-\frac{p-1}{4}\left(\tau-t_{1}\right)} d \tau .
$$

It is obvious that $J\left(t_{1}\right)=1>0$, and

$$
\begin{aligned}
J(t) & \leq 1-\frac{M_{1}(p-1)}{4} H^{\frac{p-1}{4}}\left(t_{1}\right)\left(t_{1}+1\right)^{\frac{1-p}{2}} \int_{t_{1}}^{t_{1}+1} e^{-\frac{p-1}{4}\left(\tau-t_{1}\right)} d \tau \\
& =1-M_{1} H^{\frac{p-1}{4}}\left(t_{1}\right)\left(t_{1}+1\right)^{\frac{1-p}{2}}\left(1-e^{-\frac{p-1}{4}}\right) .
\end{aligned}
$$

By (3.7), we can take $t_{1}$ sufficiently large such that

$$
H^{\frac{p-1}{4}}\left(t_{1}\right)\left(t_{1}+1\right)^{\frac{1-p}{2}} \geq \frac{1}{2}(-E(0))^{\frac{p-1}{4}} .
$$

Condition (2) of Theorem 3.1 implies

$$
J(t) \leq 0, \quad t \geq t_{1}+1
$$

Noticing the continuity of $J(t)$, we know that there is a constant $T^{*}\left(t_{1}<T^{*}<t_{1}+1\right)$ such that $J\left(T^{*}\right)=0$. Hence $S(t) \rightarrow \infty$, as $t \rightarrow T^{*}$. It follows from Lemma 3.1 that when $t \geq t_{1}$, $H(t) \geq S(t)$. Thus, $H(t) \rightarrow \infty$ as $t \rightarrow T^{*}$. Theorem 3.1 is proved.

\section{Decay rate of energy}

In this section, we are going to discuss the decay rate of energy for problem (1.1)-(1.3). We need the following lemma.

Lemma 4.1 ([11]) Suppose that $J:[0, \infty) \rightarrow[0, \infty)$ is a non-increasing function and assume that there is a constant $L>0$ such that

$$
\int_{t}^{\infty} J(s) d s \leq L J(t), \quad \forall t \geq 0
$$

Then

$$
J(t) \leq J(0) e^{1-\frac{t}{L}}, \quad \forall t \geq 0 .
$$

Theorem 4.1 Suppose that the assumptions of Theorem 2.1 hold and $2 F(s) \leq f(s)$ s. Let $u(x, t)$ be a global generalized solution for problem (1.1)-(1.3). Then we have

$$
G(t) \leq G(0) e^{1-M t}
$$

where $G(t)=\int_{\Omega}\left|\nabla w_{t}\right|^{2} d x+\int_{\Omega}|\nabla u|^{2} d x+2 \int_{\Omega} F(u) d x$. 
Lu and Wang Boundary Value Problems ( 2018) 2018:62

Page 13 of 15

Proof Recalling (3.1), we derive

$$
\begin{aligned}
G(t)= & \int_{\Omega}\left|\nabla w_{t}\right|^{2} d x+\int_{\Omega}|\nabla u|^{2} d x+2 \int_{\Omega} F(u) d x \\
= & \left\|\nabla w_{t}(0)\right\|^{2}+\|\nabla \varphi\|^{2}+2 \int_{\Omega} F(\varphi(x)) d x-2 k \int_{0}^{t} \int_{\Omega}\left|u_{\tau}(x, \tau)\right|^{2} d x d \tau \\
& -2 \int_{0}^{t} \int_{\Omega}\left|\nabla w_{\tau}(x, \tau)\right|^{2} d x d \tau .
\end{aligned}
$$

A simple computation gives, for any $0 \leq t_{1} \leq t_{2}<\infty$,

$$
\begin{aligned}
G\left(t_{1}\right)-G\left(t_{2}\right)= & 2 k \int_{0}^{t_{2}} \int_{\Omega}\left|u_{t}(x, t)\right|^{2} d x d t+2 \int_{0}^{t_{2}} \int_{\Omega}\left|\nabla w_{t}(x, t)\right|^{2} d x d t \\
& -\left(2 k \int_{0}^{t_{1}} \int_{\Omega}\left|u_{t}(x, t)\right|^{2} d x d t+2 \int_{0}^{t_{1}} \int_{\Omega}\left|\nabla w_{t}(x, t)\right|^{2} d x d t\right) \\
= & 2 k \int_{t_{1}}^{t_{2}} \int_{\Omega}\left|u_{t}(x, t)\right|^{2} d x d t+2 \int_{t_{1}}^{t_{2}} \int_{\Omega}\left|\nabla w_{t}(x, t)\right|^{2} d x d t,
\end{aligned}
$$

which shows that $G(t)$ is non-increasing.

Multiplying (1.1) by $w(x, t)$, integrating over $\left(t_{1}, t_{2}\right) \times \Omega$, and integrating by parts, we have

$$
\begin{aligned}
& -\int_{t_{1}}^{t_{2}} \int_{\Omega}\left(\left|\nabla w_{t}\right|^{2}+|\nabla u|^{2}+2 F(u)\right) d x d t \\
& =-\left.\left(\int_{\Omega} \nabla w \nabla w_{t} d x\right)\right|_{t_{1}} ^{t_{2}}-\left.\left(\int_{\Omega} u^{2} d x\right)\right|_{t_{1}} ^{t_{2}}-\left.\left(\int_{\Omega}|\nabla w|^{2} d x\right)\right|_{t_{1}} ^{t_{2}} \\
& \quad+\int_{t_{1}}^{t_{2}} \int_{\Omega}(2 F(u)-f(u) u) d x d t
\end{aligned}
$$

which implies

$$
\begin{aligned}
\int_{t_{1}}^{t_{2}} G(t) d t= & 2 \int_{t_{1}}^{t_{2}} \int_{\Omega}\left|\nabla w_{t}\right|^{2} d x d t \\
& -\left.\left(\int_{\Omega} \nabla w \nabla w_{t} d x\right)\right|_{t_{1}} ^{t_{2}}-\left.\left(\int_{\Omega} u^{2} d x\right)\right|_{t_{1}} ^{t_{2}}-\left.\left(\int_{\Omega}|\nabla w|^{2} d x\right)\right|_{t_{1}} ^{t_{2}} \\
& +\int_{t_{1}}^{t_{2}} \int_{\Omega}(2 F(u)-f(u) u) d x
\end{aligned}
$$

Recalling the assumption $f(s) s \leq 2 F(s)$, we know

$$
\begin{aligned}
\int_{t_{1}}^{t_{2}} G(t) d t \leq & 2 \int_{t_{1}}^{t_{2}} \int_{\Omega}\left|\nabla w_{t}\right|^{2} d x d t \\
& -\left.\left(\int_{\Omega} \nabla w \nabla w_{t} d x\right)\right|_{t_{1}} ^{t_{2}}-\left.\left(\int_{\Omega} u^{2} d x\right)\right|_{t_{1}} ^{t_{2}}-\left.\left(\int_{\Omega}|\nabla w|^{2} d x\right)\right|_{t_{1}} ^{t_{2}}
\end{aligned}
$$


Using the Poincaré inequality, we obtain

$$
\begin{aligned}
& \int_{\Omega} u^{2} \leq C_{*} \int_{\Omega}|\nabla u|^{2} d x \leq C_{*} G(t) \\
& \int_{\Omega}|\nabla w|^{2} \leq C_{*} \int_{\Omega}|u|^{2} d x \leq C_{*}^{2} \int_{\Omega}|\nabla u|^{2} d x \leq C_{*}^{2} G(t) .
\end{aligned}
$$

The Cauchy inequality yields

$$
\left|\int_{\Omega} \nabla w \nabla w_{t} d x\right| \leq \frac{1}{2} \int_{\Omega}|\nabla w|^{2} d x+\frac{1}{2} \int_{\Omega}\left|\nabla w_{t}\right| d x \leq \frac{1}{2}\left(C_{*}^{2}+1\right) G(t) .
$$

On the other hand, by the non-increasing property of $G(t)$, we get

$$
2 \int_{t_{1}}^{t_{2}} \int_{\Omega}\left|\nabla w_{t}\right|^{2} d x d t \leq G\left(t_{1}\right)-G\left(t_{2}\right) \leq G\left(t_{1}\right)
$$

Using (4.4)-(4.7), we deduce

$$
\int_{t_{1}}^{t_{2}} G(t) d t \leq 2\left(C_{*}+3 C_{*}^{2}+2\right) G\left(t_{1}\right) \equiv \frac{1}{M} G\left(t_{1}\right) .
$$

By Lemma 4.1, we conclude that

$$
G(t) \leq G(0) e^{1-M t}, \quad \forall t \geq 0
$$

This completes the proof.

\section{Acknowledgements}

The authors would like to express their deep thanks to the referee for valuable suggestions for the revision and improvement of the manuscript.

\section{Funding}

This work is supported by the Jilin Scientific and Technological Development Program [number 20170101143JC].

\section{Abbreviations}

Not applicable.

\section{Availability of data and materials}

Not applicable.

\section{Competing interests}

The authors declare that they have no competing interests.

\section{Authors' contributions}

All authors contributed equally to the manuscript and read and approved the final manuscript.

\section{Publisher's Note}

Springer Nature remains neutral with regard to jurisdictional claims in published maps and institutional affiliations. Received: 11 March 2018 Accepted: 11 April 2018 Published online: 25 April 2018

\section{References}

1. Galenko, P.: Phase-field models with relaxation of the diffusion flux in nonequilibrium solidification of a binary system. Phys. Lett. A 287, 190-197 (2001)

2. Zheng, S., Milani, A.: Exponential attractors and inertial manifolds for singular perturbations of the Cahn-Hilliard equations. Nonlinear Anal. 57, 843-877 (2004) 
3. Zheng, S., Milani, A.: Global attractors for singular perturbations of the Cahn-Hilliard equations. J. Differ. Equ. 209, 101-139 (2005)

4. Gatti, S., Grasselli, M., Miranville, A., Pata, V:: On the hyperbolic relaxation of the one-dimensional Cahn-Hilliard equation. J. Math. Anal. Appl. 312, 230-247 (2005)

5. Gatti, S., Grasselli, M., Miranville, A., Pata, V.: Hyperbolic relaxation of the viscous Cahn-Hilliard equation in 3-D. Math. Models Methods Appl. Sci. 15, 165-198 (2005)

6. Grasselli, M., Petzeltová, H., Schimperna, G.: Asymptotic behaviour of a non-isothermal viscous Cahn-Hilliard equation with inertial term. J. Differ. Equ. 239, 38-60 (2007)

7. Chen, G., Lu, B.: The initial-boundary value problems for a class of nonlinear wave equations with damping term. J. Math. Anal. Appl. 351, 1-15 (2009)

8. Wang, Y.: Finite time blow-up and global solutions for fourth order damped wave equations. J. Math. Anal. Appl. 418, 713-733 (2014)

9. Escudero, C., Gazzola, F., Peral, I.: Global existence versus blow-up results for a fourth order parabolic PDE involving the Hessian. J. Math. Pures Appl. 103, 924-957 (2015)

10. Qu, C., Zhou, W.: Blow-up and extinction for a thin-film equation with initial-boundary value conditions. J. Math. Anal. Appl. 436, 796-809 (2016)

11. Komornik, V.: Exact Controllability and Stabilization: The Multiplier Method. Wiley, New York (1994)

\section{Submit your manuscript to a SpringerOpen ${ }^{\circ}$ journal and benefit from:}

- Convenient online submission

- Rigorous peer review

Open access: articles freely available online

- High visibility within the field

- Retaining the copyright to your article

Submit your next manuscript at $\boldsymbol{\nabla}$ springeropen.com 